\title{
S- and X-band SAR Data Fusion
}

\author{
Raffaella Guida, Su Wai Ng, Pasquale Iervolino \\ Surrey Space Centre \\ University of Surrey \\ Guildford, United Kingdom \\ r.guida@surrey.ac.uk
}

\begin{abstract}
This paper investigates the benefits deriving from introducing a wavelet-transform-based fusion framework for multi-frequency Synthetic Aperture Radar (SAR) data. A specific application is considered in the assessment of the fused classification map derived and this is the discrimination of different kinds of oil in sea. S-band and X-band datasets, concurrently acquired from the same airborne platform, have here been used. The findings suggest that fusing S-band and $X$ band SAR data does improve the oil type discrimination between crude oil and diesel oil used in the exercise, although a more quantitative analysis should be conducted in the future to measure the degree of improvement.
\end{abstract}

Index Terms-Synthetic Aperture Radar (SAR), S-band, Xband, data fusion, wavelet transform, oil discrimination, oil spill.

\section{INTRODUCTION}

In the past two decades, several Synthetic Aperture Radar (SAR) imaging systems were successfully built, launched and operated from both airborne and spaceborne platforms. Availability of these SAR systems has provided end-users with a significant amount of data in multimodal fashion, i.e. multi-frequency ( $\mathrm{P}, \mathrm{L}, \mathrm{S}, \mathrm{C}, \mathrm{X}$ and $\mathrm{Ku}$ band), multi-angle $\left(10^{\circ}-70^{\circ}\right)$, multi-polarisation $(\mathrm{HH}, \mathrm{HV}, \mathrm{VV}$ and $\mathrm{VH})$ and multi-resolution data (from tens of centimetres to hundred kilometres). In addition to existing operational systems, there are more SAR systems that are currently under development. These new SAR systems will not only increase the amount of data available to the users, but also possibly add new dimensionality for new applications via new capabilities onboard the system, e.g. addition of new operating frequencies such as the S-band. While it is certainly useful to investigate or study these data alone, there is an increasing interest and effort to fuse multi-platform data with the aims to obtain more information from the fused image than looking at individual sensor data alone. However, most of these efforts have been focused on fusing the image from different sources of sensor, e.g. fusing data from optical imaging systems with SAR data or with LIDAR data. There is instead little or limited study on fusing the multi-frequency SAR data alone.

This paper investigates the possibility of multi-frequency SAR data fusion with particular reference to oil spill discrimination. It contains a brief description of an oil on water exercise (Section 2), conducted on 25th June 2014 south-west off the Isle of Wight, within the Satellite Application Catapult
SAR demonstrator program, and presents a theoretical data fusion framework based on wavelet transforms developed and validated with $\mathrm{S}$ - and $\mathrm{X}$-band SAR datasets acquired during the exercise (Section 3 ). The findings from the study are discussed in Section 4 and finally conclusions are driven.

\section{OIL ON WATER EXERCISE}

The controlled oil spill experiment was conducted southwest off the Isle of Wight on 25 June 2014 to allow image acquisition of oil slicks via the Airbus Defence and Space AirSAR demonstrator flying on a NERC platform.

Two types of oil with different properties were released, i.e. Weald Basin Crude and Marine Diesel which were discharged about, respectively, 30 minutes and 23 minutes prior the SAR acquisition from the demonstrator. Tasking of Radarsat-2 SAR satellite was also planned in order to acquire spaceborne images simultaneously over the oil spill site and finally get a multi-platform (spaceborne and airborne), multi-frequency (Sand X-band from airborne, C-band from spaceborne) fully polarimetric SAR dataset for research purposes. In this paper only the airborne acquisition was used with the purpose to simplify the coregistration of datasets and allow to test a SAR fusion concept on a controlled scenario.

With the purpose of monitoring the exercise from the beginning of the discharge till the completion of the clean-up operations, twenty AirSAR image strips were acquired in total at different stages of the exercise. The two datasets considered here were taken prior the beginning of the clean-up operation, which affected the existing separation between the two different oil spills. Information on wind speed and direction was extracted from meteorological data measurement from the instrumentation onboard one of the vessels in the area. The wind speed was, respectively, about 4 and 9 knots (corresponding to $2.06 \mathrm{~ms}^{-1}$ and $4.63 \mathrm{~ms}^{-1}$ ) during the imaging time of the two SAR strips here considered. This wind speed just exceeds the value of $2 \mathrm{~ms}^{-1}$, which is the typically required lower limit of wind speed for oil slicks dark spot detection in SAR images [1].

\section{SAR DATA FUSION}

The overall plan for the SAR image fusion is shown in Figure 1, which comprises of three main stages of processing, namely image pre-processing, fusion operation and accuracy assessment via case study validation. The pre-processing stage 


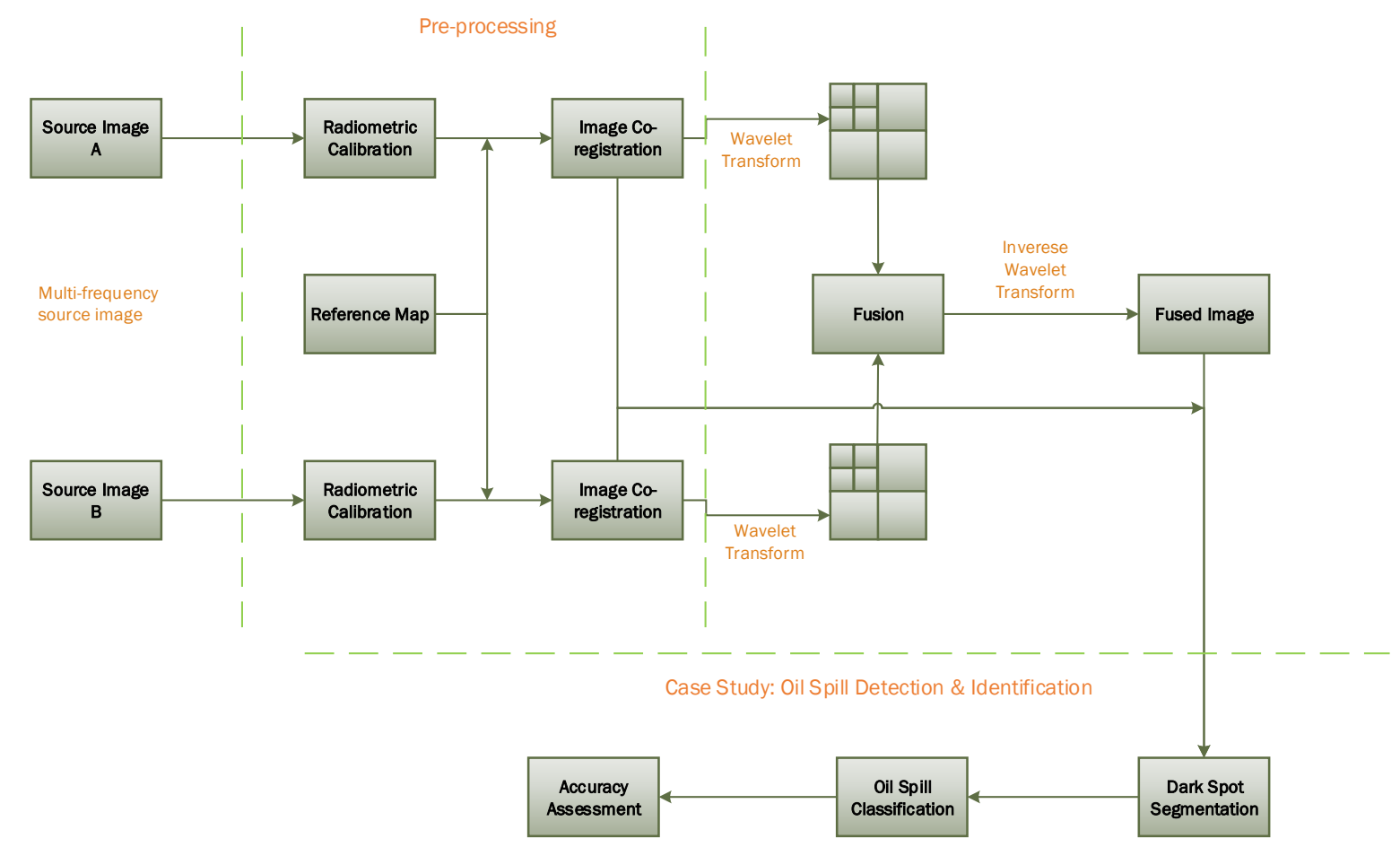

Fig. 1. SAR data fusion using wavelet transform approach

includes a block of radiometric calibration which essentially deals with local incidence angle normalisation and speckle noise reduction, as the AirSAR images provided had already been corrected for systematic errors. The purpose of image coregistration, instead, is to "align" the images to be fused so that the same pixels in all images represent the same feature on the ground. As the $\mathrm{X}$ and S-band AirSAR system is acquiring images of the ground simultaneously from the same platform, the geometry of both images is assumed to be identical with no difference in geometric distortions. However, C-band SAR image from Radarsat- 2 had totally different viewing geometry and altitude during image acquisition compared to the AirSAR images. Co-registration was therefore necessary to align the Radarsat-2 SAR image to a common spatial frame with AirSAR images.

The wavelet transform is a multi-resolution decomposition in which, during the process of signal decomposition into subcomponents and its recovering from the sub-components no information is lost. Multi-resolution analysis (MRA) allows one to decompose the signal of interest into many subcomponents at different resolutions for independent operations at each level. In this way an input signal $\mathrm{S}^{0}$ can be decomposed into an approximation and a detail signal, $\mathrm{A}^{1}$ and $\mathrm{D}^{1}$. The Approximation signal is a low resolution or coarse approximation of $\mathrm{S}^{0}$, while the detail signal constitutes the fine details or high frequency component that is discarded during simplification of $\mathrm{S}^{0}$ to $\mathrm{A}^{1}$. In real world applications, the decomposition does not stop at the first level and will further separate $A^{1}$ into $A^{2}$ and $D^{2}$ and subsequently $A^{k}$ into $A^{k+1}$ and
$\mathrm{D}^{\mathrm{k}+1}$ in multi-level decomposition. A wavelet transform has to satisfy the condition of perfect reconstruction of multiresolution analysis but also guarantee that the decomposition is not redundant.

In the fusion scheme in Fig. 1, Daubechies wavelets were considered in conjunction with a choose-max or maximum selection rule for the coefficients combining method, a popular choice based on the fact that the largest coefficient value typically reflects salient features in the images such as edges, lines and region boundaries. Alternatively, a choose-mean or average selection rule can be applied and an example is given in the discussion of results. The wavelet coefficients with largest salient values were then selected for integration into the new fused image and, after that, the following fusion rule was applied: mathematically, if $\mathrm{Z}$ is the fused image, $\mathrm{X}$ and $\mathrm{Y}$ are the input images, $D_{I}$ and $A_{I}$ are multi-level decomposition representation and activity level of image $I$, respectively, then this can be described as $\mathrm{D}_{\mathrm{z}}(\mathrm{p})=\mathrm{D}_{\mathrm{i}}(\mathrm{p})$, where $\mathrm{i}=\mathrm{X}$ or $\mathrm{Y}$ depending on which source image satisfies,

$$
\mathrm{A}_{\mathrm{i}}(\mathrm{p})=\max \left(\mathrm{A}_{\mathrm{x}}(\mathrm{p}), \mathrm{A}_{\mathrm{y}}(\mathrm{p})\right)
$$

The main objective of dark spot detection block is to identify and isolate dark spots formed in the SAR image, because of the presence of oil slicks, via segmentation and thresholding process. In this study, a region-based segmentation approach called watershed transform is used to break down the image into various disjoint regions which are internally homogeneous, [2]. 


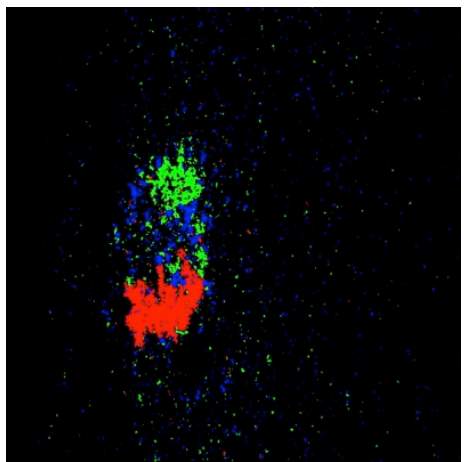

$\mathrm{S}$ band

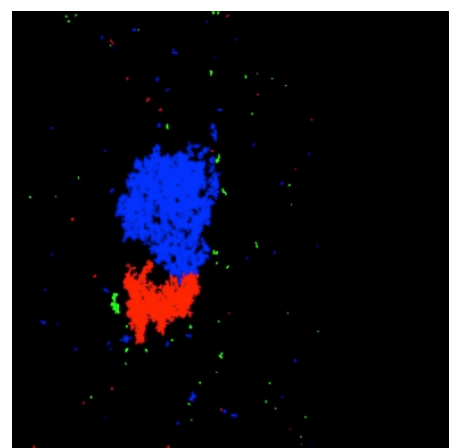

Db1 1 max

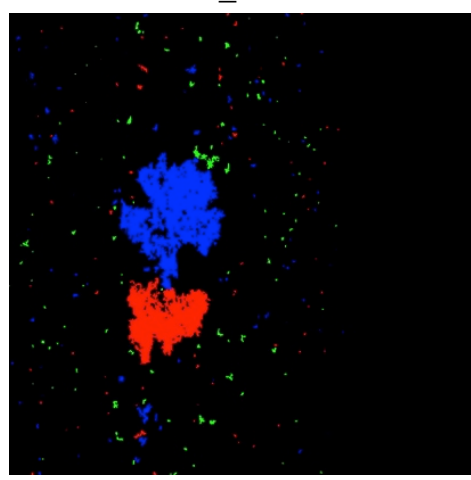

Db2_3 max

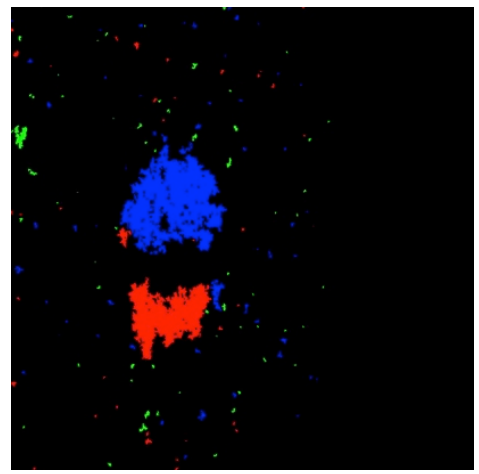

$\mathrm{X}$ band

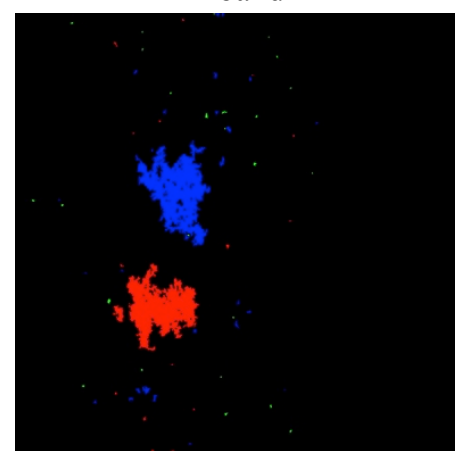

Db2 1 max

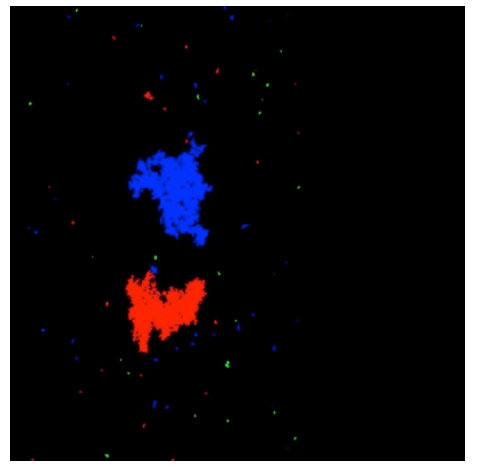

Db5_1 max
Legend
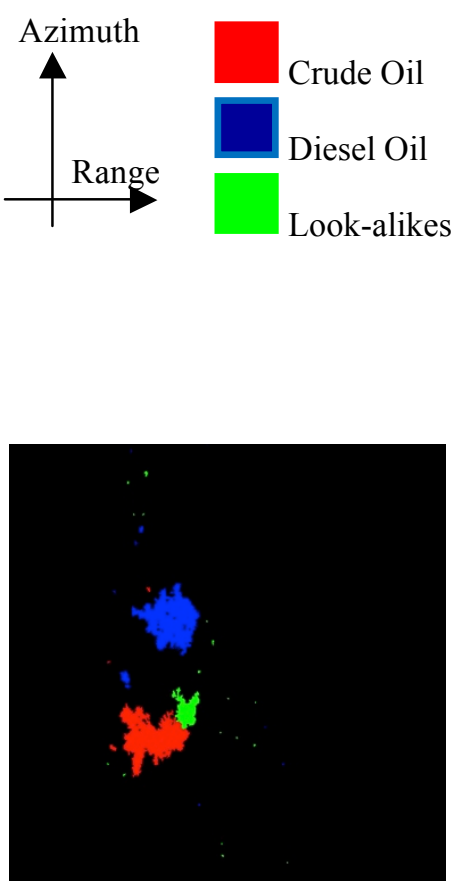

Db2_1 mean

Fig. 2. Unsupervised classification result of HV image into three classes.

Considering the lack of a-priori information and the needs for automatic approach in typical oil spill identification application, unsupervised oil spill classification is suggested to classify the segmentation image. More specifically, classic unsupervised k-mean classification has been identified as the classifier tool in oil spill application, [3].

Finally, a quantitative accuracy assessment is performed based on relative mean bias and universal image quality index (UIQI) to evaluate the general quality of the fused images. A qualitative assessment of the improvement in oil spill discrimination coming from the fusion of S- and X- band SAR data is also performed and is presented in the next section.

\section{RESULTS}

As discussed in Section 3, Daubechies wavelet transform and choose-max fusion rule is proposed for fusing $\mathrm{S}$ and $\mathrm{X}$ band AirSAR images relevant to the same strips. There are two parameters that control the performance of Daubechies wavelet, i.e. number of vanishing moments and decomposition levels. The higher the number of vanishing moments, the more sparse the representation of the image is. However, such wavelet is less systematic and irregular which in turn generates more image artefacts [4]. Meanwhile, the required level of image decomposition depends on the amount of fine details present in the original image, i.e. the amount of horizontal, vertical and diagonal edge information. The input images are practically very low details image with limited number of high spatial frequency features. Thus, Daubechies 
WT with two vanishing moments and one level of decomposition is the main transform adopted in this study. Other numbers of vanishing moments and decomposition levels, as well as general weighted average rule, have been applied and here the latter is incorporated for comparison. In the following, the abbreviation $\mathrm{Dbi} j \max$ refers to Daubechies wavelet transform with vanishing moment $i$ and decomposition level $j$, together with choose-max fusion rule, in contrast to $\mathrm{Db} i j$ mean where choose-mean fusion rule has been applied.

Unsupervised k-mean classification was carried out on $\mathrm{HH}, \mathrm{VV}$ and HV images. Three classes were selected in the classification step in an attempt to represents crude oil, diesel oil and look-alikes. Overall, fused images give the best classification result with both crude oil and diesel oil clearly discriminated in most cases, except for VV images. HV images, in Fig.2, show more promising classification results in differentiating between crude oil and diesel oil if compared to $\mathrm{HH}$ and VV images. Every HV image except S-band and Db2_1 mean images, succeeds in classifying crude oil and diesel oil into two distinct classes. The amount of tiny fragmented look-alikes segments surrounding the actual oil slicks are generally lesser in fused images as compared to Xband. For $\mathrm{HH}$ images, $\mathrm{Db} 2 \_1$ mean and $\mathrm{Db} 2 \_3$ max fused results are poor but still relatively better than S-band. There is not too much difference among the X-band and Db1_1 max, Db2_1 max and Db5_1 max classification results. Lastly for VV image, Db2_1 image is the only fused image that performs relatively better than input images, but the result is still unable to distinguish crude oil and diesel oil into 2 distinct classes.

As can be expected, average mean fusion rule fail to improve classification accuracy if one of the input image is not of good quality (the S-band image in this case). The poor quality of input data may be due to different factors such as, for example, the wind speed just above $2 \mathrm{~ms}^{-1}$ during the acquisition of AirSAR imagery, which is the reported lower limit for dark spot formation, and the weathering of diesel oil slicks causing the discrepancy in the observed contrast.

On the other hand, choose-max fusion rule also relies on the quality of both input images but most of the time the results achieved are as good as the best of two input images if not better than. Combination between Db2_1 WT and choosemax fusion rule yields the best performance among the variety of image fusion combinations tested in this case study.

\section{CONCLUSIONS}

This paper has presented some of the results of a larger and more comprehensive research project on feasibility and effectiveness of SAR data fusion. The fusion experiment carried out in this study is unique because based on the availability of multi-frequency SAR data concurrently acquired on an ad-hoc designed oil on water exercise to study oil spill detection and identification. SAR data fusion has been successfully performed and based on the available AirSAR Sband and X-band imagery finding that the fused results in general improve the differentiation of crude oil and diesel oil. Combination between Daubechies wavelet transform with 2 vanishing moments and 1 decomposition level as well as choose-max fusion rule yields the best classification result. But the improvement demonstrated is not significant due to poor contrast accounted with diesel oil dark spot formation. A comparison later conducted with quite concurrent RadarSat-2 C-band images indicate that the problem only exists in AirSAR data. It should also be noted that the findings are based on visual inspection and qualitative evaluation only and conclusive remarks cannot be drawn unless quantitative assessment is performed.

The ground truth data about the estimated extent of the oil slicks area is very useful data for quantitative assessment which represents indeed the next step in this study. In this sense, an attempt will be made in the future using also infrared and optical imagery acquired from a balloon during the overall exercise.

The authors are now working on the design of the next exercise in which different alternative options are under consideration and regard the type of controlled oil discharge and different data fusion approaches based on contourlet transform and sparse representation based technique. Likewise, different segmentation and classification techniques should also be tested. Using oil spill detection and identification represents a limit case to validate the SAR data fusion theoretical framework. There are mainly two reasons: on one side, it is very difficult to find good Ground Control Points to coregister SAR ocean images, on the other salient features in oil spill image, i.e. the contrast between oil slicks dark spot and background sea water, are not always adequate. Majority of image fusion techniques make use of the salient features to enhance the output. Some scenes in the SAR image are naturally rich with plenty of vertical, horizontal or diagonal details, such as urban area or agricultural land. Application related to these areas can be selected for future case study.

\section{ACKNOWLEDGMENT}

The authors thank the Satellite Application Catapult for making the data from the oil on water exercise available for this study and Airbus Defence and Space and NERC for providing, respectively, the SAR demonstrator and platform used in the exercise.

\section{REFERENCES}

[1] C. Brekke and A. H. S. Solberg. Oil spill detection by satellite remote sensing. Remote Sens. Environ. 95(1), pp. 1-13. 2005.

[2] J. B. T. M. Roerdink and A. Meijster. The watershed transform: Definitions, algorithms and parallelization strategies. Fundam.Inf. 41(1,2), pp. 187-228. 2000.

[3] R. A. Schowengerdt. "Chapter 7: Correction and calibration," in Remote Sensing: Models and Methods for Image Processing (3rd edition ed.), 2006.

[4] M. Misiti, Y. Misiti, G. Oppenheim and J. Poggi. Wavelets and their Applications 2007330. 